\title{
ON THE RELATION BETWEEN \\ THE SINGULAR AND THE WEAK COUPLING LIMITS
}

\author{
L. Accardi*, A. Frigerio**, Y.G.Lu*** \\ * Dipartimento di Matematica, Università di Roma II \\ * Dipartimento di Scienza dell'Informazione, Università di Milano \\ *** Dipartimento di Matematica, Università di Roma II, On leave of absence from Beijng Normal Uniersity
}

\begin{abstract}
After having recalled some definitions concerning quantum stochastic processes and in particular quantum Brownian motions, a general scheme is introduced which allows a unified approach to the weak coupling and the singular coupling limits. The analogies and differences between the two are discussed. The main difference consists in the fact that, in the singular coupling limit, the use of a Hamiltonian unbounded below seems to be unavoidable, while this is not the case for the weak coupling limit.
\end{abstract}

\section{1. Introduction}

In this section we introduce a general abstract scheme which allows a unified treatment of the singular and the weak coupling limit, in any gaussian reference state of the reservoir (Fock, finite temperature, squeezing, ...).

The singular coupling limit for open quantum systems has been widely studied in the physical literature. A first attempt towards a rigorous treatment is due to Hepp and Lieb ([1]) and was pushed further by Gorini and Kossakowski ([2]), Frigerio and Gorini ([3]). Its connection with the weak coupling limit has been explained by Palmer ([4]) and studied also by Spohn in his review paper [5]. The convergence of multi-time correlation functions has been studied by Dümcke ([6]) and by Frigerio and Gorini ([7]). 
The basic idea of the singular coupling limit is as follows. Consider a quantum system $S$ coupled to a quasi-free boson or fermion reservoir $R$, with total Hamiltonian

$$
H_{\lambda}=H_{S} \otimes 1_{R}+1_{S} \otimes H_{R}+V_{\lambda}
$$

This means that two Hilbert spaces $\mathcal{H}_{S}, \mathcal{H}_{R}$ are given, interpreted respectively as the Hilbert space of the sytem $S$ and of the reservoir $R$. It is moreover assumed that $\mathcal{H}_{R}$ is obtained by quasi-free (gaussian) second quantization from a 1 -particle Hilbert space $\mathcal{H}_{1}$, with creation and annihilation operators denoted $a^{+}(f), a(g)\left(f, g \in H_{1}\right)$ and that the reservoir Hamiltonian $H_{R}$ is quasi-free, i.e.

$$
e^{i H_{R} t} a(f) e^{-i H_{R} t}=a\left(S_{t} f\right)
$$

$S_{t}=\exp \left(i H_{1} t\right)$ being a strongly continuous one-parameter group on the Hilbert space $\mathcal{H}_{1}$ of test functions. We assume that the interaction has the form

$$
V_{\lambda}=\frac{1}{i}\left[D \otimes a^{+}\left(g^{\lambda}\right)-D^{+} \otimes a\left(g^{\lambda}\right)\right]
$$

or, more generally, is a sum of terms of this kind,

$$
V_{\lambda}=\frac{1}{i} u m_{j}\left[D \otimes a^{+}\left(g_{j}^{\lambda}\right)-D^{+} \otimes a\left(g_{j}^{\lambda}\right)\right]
$$

with $\left\langle g_{j}^{\lambda}, S_{t} g_{k}^{\lambda}\right\rangle=0$ for $j \neq k$ and for all $t$.

Let $Q \geq 1$ be an operator on $H_{1}$ with domain $\mathrm{D}(Q) ; \mathcal{I}$ be a family of bounded closed intervals in $\mathbf{R} ; K$ be a set and let, for each $\lambda>0,[S, T] \in \mathcal{I}$ and $f \in K$, be given a vector $f_{t}^{\lambda} \in D(Q) \subseteq H_{1}$ so that, for $\varepsilon=0,1\left(Q^{0}=1\right.$; $\left.Q^{1}=Q\right)$, there exist the limits

$$
\lim _{\lambda \rightarrow 0}\left\langle\int_{S}^{T} d s f_{s}^{\lambda}, Q^{\varepsilon} \int_{S^{\prime}}^{T^{\prime}} g_{u}^{\lambda} d u\right\rangle=\left\langle\chi_{[S, T]}, \chi_{\left[S^{\prime}, T^{\prime}\right]}\right\rangle_{L^{2}(\mathbf{R})} \cdot(f \mid g)_{Q^{\varepsilon}}
$$

where $(\cdot \mid \cdot)_{Q^{\varepsilon}}$ is a positive kernel on $K$ (not necessarily positive definite).

For each $\lambda>0$ we shall denote by $F_{\lambda}$ the family of vectors $f_{t}^{\lambda}$, for $t \in \mathbf{R}$ and $f \in K$. The positivity of the kernel $(\cdot \mid \cdot)$ follows from (1.1); it can happen that the kernel is degenerate.

In the following the notation $K$ will be also used to denote the pre-Hilbert space $(K / \operatorname{Ker}((\cdot \mid \cdot)),(\cdot \mid \cdot))$.

In order to state our basic result we shall introduce some definitions and notations: 
$\underline{D E F I N I T I O N}$ Let $\mathcal{K}$ be a Hilbert space, $\mathrm{T}$ an inteval in $\mathbf{R}$. Let $Q \geq 1$ be a self-adjoint operator on $\mathcal{K}$ with domain $\mathcal{D}(Q)$ and let

$$
\left\{\mathcal{H}_{Q}, \pi_{Q}, \Psi_{Q}\right\}
$$

denote the GNS representation of the CCR over $L^{2}(T, d t ; \mathcal{K})$ with respect to the quasi-free state $\varphi_{Q}$ on $W\left(L^{2}(T, d t ; \mathcal{K})\right)$ characterized by

$$
\varphi_{Q}(W(\xi))=e^{-\frac{1}{2}<\xi, 1 \otimes Q \xi>} \quad ; \quad \xi \in \mathcal{D}(Q) \subset L^{2}(T, d t ; \mathcal{K})
$$

The stochastic process, in the sense of [10]

$$
\left\{\mathcal{H}_{Q}, A\left(\chi_{(s, t]} \otimes f\right), A^{+}\left(\chi_{(s, t]} \otimes f\right) ;(s, t] \subseteq T, f \in \mathcal{K}\right\}
$$

on the domain of coherent vectors, where $A(\cdot), A^{+}(\cdot)$ denote respectively the annihilation and creation fields, is called the Boson Q-Quantum Brownian Motion on $L^{2}(T, d t ; \mathcal{K})$. The 1-Quantum Brownian Motion will be called the Fock Brownian Motion . In this case the space $\mathcal{H}_{Q}=\mathcal{H}_{1}$ is isomorphic to the Fock space over $L^{2}(T, d t ; \mathcal{K})$ and denoted $\Gamma\left(L^{2}(T, d t ; \mathcal{K})\right)$.

$\operatorname{THEOREM}(1.1) \quad 1$ For any $n \in \mathbf{N}, f_{1}, \cdots, f_{n} \in K$, as $\lambda \rightarrow 0$ the quantity

$$
\left\langle\Phi_{Q}, W_{Q}\left(x_{1} \int_{S_{1}}^{T_{1}} f_{1, t_{1}}^{\lambda} d t_{1}\right) \cdots W_{Q}\left(x_{n} \int_{S_{n}}^{T_{n}} f_{n}^{\lambda} t_{n} d t_{n}\right) \Phi_{Q}\right\rangle
$$

converges, uniformly for $x_{1}, \cdots, x_{n}$ in a bounded set of $\mathbf{R}$, to

$$
\left\langle\Psi, W\left(\chi_{\left[S_{1}, T_{1}\right]} \otimes f_{1}\right) \cdots W\left(\chi_{\left[S_{n}, T_{n}\right]} \otimes f_{n}\right) \Psi\right\rangle
$$

where $\Psi$ is the cyclic vector of the $\mathrm{QBM}$ on $L^{2}\left(\mathbf{R} ; K_{Q}\right)$ with variance $(f \mid g)_{Q}$.

EXAMPLE 1 (the weak coupling limit (WCL) with linear interaction) We take $S \subseteq H_{1}$ such that

$$
\int\left|\left\langle f, S_{t} g\right\rangle\right| d t<+\infty, \quad \forall f, g \in K
$$

(in all examples $K$ is a dense subspace of $H_{1}$ ) and define

$$
f_{t}^{\lambda}:=\frac{1}{\lambda} S_{t / \lambda^{2}} f
$$


Then

$$
\begin{aligned}
& \left\langle\int_{S}^{T} d s f_{s}^{\lambda}, \int_{S^{\prime}}^{T^{\prime}} d u g_{u}^{\lambda}\right\rangle=\frac{1}{\lambda^{2}}\left\langle\int_{S}^{T} d t S_{t / \lambda^{2}} f, \int_{S^{\prime}}^{T^{\prime}} d u S_{u / \lambda^{2}} g\right\rangle= \\
= & \frac{1}{\lambda^{2}} \int_{S}^{T} d t \int_{S^{\prime}}^{T^{\prime}} d u\left\langle f, S_{(u-t) / \lambda^{2}} g\right\rangle=\int_{S}^{T} d t \int_{\left(S^{\prime}-t\right) / \lambda^{2}}^{\left(T^{\prime}-t\right) / \lambda^{2}}\left\langle f, S_{v} g\right\rangle
\end{aligned}
$$

Thus (1.1) is true with $\varepsilon=0$. If $S_{t}^{-1} Q S_{t}=Q$ then (1.1) is true also with $\varepsilon=1 . \underline{E X A M P L E 2}$ (the singular coupling limit (SCL))

Another general scheme to construct families $\left(f_{t}^{\lambda}\right)$ is the following.

Let $K$ be a set and let the functions $f_{t}^{\lambda}$ have the form

$$
f_{t}^{\lambda}=S_{t} f^{\lambda}
$$

where $\lambda \in \mathbf{R} \mapsto f^{\lambda} \in H_{1}$ is a measurable map and for any pair of such maps $f^{\lambda}, g^{\lambda}$ there exists an integrable function $G_{f, g} \in L^{1}(\mathbf{R})$ such that

$$
\lim _{\lambda \rightarrow 0}\left\langle f^{\lambda}, S_{t} g^{\lambda}\right\rangle=\hat{G}_{f, g}(0) \operatorname{delta}(t)
$$

in the sense of distributions on $\mathbf{R}$. Here as usual $\hat{G}$ denotes the Fourier transform of a function $G$

$$
\hat{G}(\omega):=\int_{-\infty}^{+\infty} e^{-i \omega t} G(t) d t \quad(\omega \in \mathbf{R})
$$

Notice that condition (2 is surely satisfied if

$$
\int e^{-i \omega t}\left\langle f^{\lambda}, S_{t} g^{\lambda}\right\rangle d t=\hat{G}_{f, g}\left(\lambda^{2} \omega\right)
$$

In fact, if $G_{\lambda}$ is a family of functions such that

$$
\hat{G}_{\lambda}(\omega)=\hat{G}\left(\lambda^{2} \omega\right)
$$

then, for any smooth function $\varphi$ :

$$
\begin{aligned}
& \int \varphi(t) G_{\lambda}(t) d t=\int \varphi(t) d t \int e^{i t \omega} \hat{G}\left(\lambda^{2} \omega\right) d \omega=\frac{1}{\lambda^{2}} \int \varphi(t) d t \int e^{i t \omega / \lambda^{2}} \hat{G}(\omega) d \omega= \\
& =\int \varphi\left(\lambda^{2} t\right) d t \int e^{i t \omega} \hat{G}(\omega) d \omega=\int \varphi\left(\lambda^{2} t\right) d t G(t)=\varphi(0) \cdot \int G(t) d t=\varphi(0) \hat{G}(0)
\end{aligned}
$$


i.e. $\lim _{\lambda \rightarrow 0} G_{\lambda}=\hat{G}(0)$ delta in the sense of distributions.

In the papers $[1,2,3]$, the singular coupling scheme described above is realized by choosing:

$$
\begin{gathered}
H_{1}=L^{2}(\mathbf{R}, d \omega) \\
g^{\lambda}(\omega)=g\left(\lambda^{2} \omega\right) \quad ; \quad \omega \in \mathbf{R} \quad, \quad g \in H_{1} \\
\left(S_{t} g\right)(\omega)=e^{i \omega t} g(\omega) \quad ; \quad \omega \in \mathbf{R} \quad, \quad g \in H_{1}
\end{gathered}
$$

whence it follows that, if $g$ is continuous in zero, then:

$\hat{G}_{\lambda}(\omega)=\int_{-\infty}^{+\infty} e^{-i \omega t} \int_{-\infty}^{+\infty} e^{i \omega^{\prime} t}\left|g\left(\lambda^{2} \omega^{\prime}\right)\right|^{2} d \omega^{\prime} d t=\int_{-\infty}^{+\infty} 2 \pi \delta\left(\omega-\omega^{\prime}\right)\left|g\left(\lambda^{2} \omega^{\prime}\right)\right|^{2} d \omega^{\prime}=2 \pi\left|g\left(\lambda^{2} \omega\right)\right|^{2}$

i.e.

$$
\lim _{\lambda \rightarrow 0}\left\langle g^{\lambda}, S_{t} g^{\lambda}\right\rangle=2 \pi|g(0)|^{2} \operatorname{delta}(t)
$$

By polarization it follows that condition (2) is satisfied with $\hat{G}_{f, g}(\omega)=$ $2 \pi \overline{f(\omega)} g(\omega)$ (in $[2,3]$ the parameter $\lambda^{2}$ was called $\varepsilon$ ).

It is not really necessary that $\mathcal{H}_{1}=L^{2}(\mathbf{R}, d \omega)$; it may well be $L^{2}(I, d \omega)$ where $I$ is any interval. However this interval must contain 0 in its interior if one wishes $g(0) \neq 0$ the non triviality of the limit (8).

Notice however that, if in this construction one wants to interpret $H_{1}$ as the 1-particle space of a quantum system and $S_{t}$ as its evolution, then (1.4c) gives the form of $S_{t}$ in energy representation and shows that the generator of $S_{t}$ cannot have spectrum bounded from below.

Gorini, Kossakowski and Frigerio choose the function g to be a gaussian, i.e. the set $K$ consists of a single number 1 and

$$
f_{t}^{\lambda}(\omega)=e^{-\left(\lambda^{2} \omega\right)^{2} / 8-i \omega t} ; \quad \omega \in \mathbf{R}
$$

then

$$
\begin{gathered}
\left\langle\int_{S}^{T} d t f_{t}^{\lambda}, \int_{S^{\prime}}^{T^{\prime}} d u f_{u}^{\lambda}\right\rangle=\int_{S}^{T} d t \int_{S^{\prime}}^{T^{\prime}} d s \int_{\mathbf{R}} d \omega e^{-\lambda^{2} \omega^{2} / 4} e^{i \omega(t-s)}= \\
=\int_{S}^{T} d t \int_{S^{\prime}}^{T^{\prime}} d s \frac{e^{-(t-s)^{2} / \lambda^{2}}}{\left(2 \pi \lambda^{2}\right)^{1 / 2}}=\int_{S}^{T} d t \int_{\left(S^{\prime}-t\right) / \lambda^{2}}^{\left(T^{\prime}-t\right) / \lambda^{2}} d v \frac{e^{-v^{2}}}{\pi^{1 / 2}} \longrightarrow\left\langle\chi_{[S, T]}, \chi_{\left[S^{\prime}, T^{\prime}\right]}\right\rangle
\end{gathered}
$$

Hence (1) with $\varepsilon=0$ (Fock case) is satisfied. Also with $\varepsilon=1$, (1) is satisfied if $Q$ commutes with translations. 
A variant of the Gorini-Kossakowski case can be obtained by considering

$$
f_{t}^{\lambda}(\omega)=\frac{1}{\lambda} e^{-\left(\omega-\omega_{0}\right)^{2} / 8 \lambda^{2}} e^{i\left(\omega-\omega_{0}\right) t / \lambda^{2}}, \quad \omega \in \mathbf{R}_{+}
$$

then

$$
\begin{gathered}
\left\langle\int_{S}^{T} d s f_{s}^{\lambda}, \int_{S^{\prime}}^{T^{\prime}} d t f_{t}^{\lambda}\right\rangle= \\
=\int_{S}^{T} d s \int_{S^{\prime}}^{T^{\prime}} d t \int_{0}^{\infty} d \omega \lambda^{-2} e^{-\left(\omega-\omega_{0}\right)^{2} / 4 \lambda^{2}} e^{i\left(\omega-\omega_{0}\right)(s-t) / \lambda^{2}} \\
=\int_{S}^{T} d s \int_{S^{\prime}}^{T^{\prime}} d t \int_{-\omega_{0}}^{\infty} d \omega \lambda^{-2} e^{-\omega^{2} / 4 \lambda^{2}} e^{i \omega(s-t) / \lambda^{2}} \\
=\int_{S}^{T} d s \int_{S^{\prime}}^{T^{\prime}} d t \int_{-\omega_{0} / \lambda^{2}}^{\infty} d x e^{-\lambda^{2} x^{2} / 4} e^{i x(s-t)} \\
=\int_{S}^{T} d s \int_{S^{\prime}}^{T^{\prime}} d t \int_{-\infty}^{+\infty} d x e^{-\lambda^{2} x^{2} / 4} e^{i x(s-t)}+O\left(e^{-\omega_{0}^{2} / \lambda^{2}}\right) \\
=\int_{S}^{T} d s \int_{S^{\prime}}^{T^{\prime}} d t \frac{e^{-(s-t)^{2} / \lambda^{2}}}{\left(\pi \lambda^{2}\right)^{1 / 2}} \rightarrow\left\langle\chi_{[S, T]}, \chi_{\left[S^{\prime}, T^{\prime}\right]}\right\rangle
\end{gathered}
$$

We can interpret the space to which the $f_{z}^{\lambda}$ belong as the 1-particle space of a system with Hamiltonian $H-\omega_{0}$ in the spectral representation for $H$.

The choice (9) corresponds to a mixed SCL and WCL situation:

- the SCL part is $\frac{1}{\lambda} e^{-\left(\omega-\omega_{0}\right)^{2} / 8 \lambda^{2}}$

- the WCL part is $e^{-i\left(\omega-\omega_{0}\right) t / \lambda^{2}}$

This model is better than the GKF because here the Hamiltonian is bounded below and the shift in frequency is physically motivated (RWA).

\section{Convergence of the Wave operator}

In the assumptions of the section 1) consider the iterated series

$$
U_{t}^{(\lambda)}=u m_{n=0}^{\infty} \int_{0}^{t} d t_{1} \operatorname{dots} \int_{0}^{t_{n-1}} d t_{n} V\left(g_{t_{1}}^{\lambda}\right) \operatorname{dot} \operatorname{V} V\left(g_{t_{n}}^{\lambda}\right)
$$

one has the following THEOREM (2.1) I If the functions $\left(f_{t}^{\lambda}\right)$ satisfy: 
1) $\left\langle f_{s}^{\lambda}, g_{t}^{\lambda}\right\rangle=\left\langle f_{0}^{\lambda}, g_{t-s}^{\lambda}\right\rangle$

2) $\forall f, g \in k$ there exists a constant $M(f, g)$ such that, for any $\lambda>0$

$$
\int_{\mathbf{R}}\left|\left\langle f_{0}^{\lambda}, g_{t}^{\lambda}\right\rangle\right| d t \leq M(f, g)<+\infty
$$

then

$\lim _{\lambda \rightarrow 0}\left\langle u \otimes \Phi\left(\int_{S_{1}}^{T_{1}} f_{1, u}^{\lambda} d u\right), U_{t}^{(\lambda)} v \otimes \Phi\left(\int_{S_{2}}^{T_{2}} f_{2, v}^{\lambda} d v\right)\right\rangle=\left\langle u \otimes \Phi\left(\chi_{[S, T]} \otimes f_{1}\right), U(t) v \otimes \Phi\left(\chi_{\left[S_{2} T_{2}\right]} \otimes f_{2}\right)\right\rangle$

where the limit $U(t)$ is solution of a quantum stochastic differential equation driven by quantum Brownian motion.

The proof of the theorem (2.1) is similar to what we have done in the section 6) of [11].

\section{$3 \quad 3$ The modified SCL as a modified WCL}

A slight modification of the singular coupling scheme, described in Section 1 , leads to a connection between the schemes of the weak and the singular coupling. This connection was discovered by Palmer ([4]) and discussed more explicitly by Spohn ([5]).

In the class of models considered by Hepp, Lieb, Frigerio, Gorini, Kossakowski, Verri, Sudarshan ..., condition (3) becomes

$$
\int_{-\infty}^{+\infty} e^{i \omega t}\left\langle g^{\lambda}, S_{t} g^{\lambda}\right\rangle d t=\hat{G}\left(\lambda^{2} \omega\right) ; g \in L^{2}(\mathbf{R})
$$

where $\hat{G}(\alpha)=\int_{-\infty}^{+\infty} e^{i \alpha u}\left\langle g, S_{u} g\right\rangle d u$.

Let us now relax this condition by admitting a $\lambda$-dependence not only in the test functions but also in the evolution $S_{t}$. More precisely, let $H_{1}$ be arbitrary, and let $S_{t}=\exp \left[i H_{1} t\right]$ be a strongly continuous one-parameter semigroup on $H_{1}$ with Lebesgue spectrum. Define

$$
g^{\lambda}=\frac{1}{\lambda} g
$$

and replace $S_{t}$ by

$$
S_{t}^{(\lambda)}:=S_{t / \lambda^{2}}
$$


Then

$$
\begin{gathered}
\int_{-\infty}^{+\infty} e^{-i \omega t}\left\langle g^{\lambda}, S_{t}^{(\lambda)} g^{\lambda}\right\rangle d t=\int_{-\infty}^{+\infty} e^{-i \omega t}\left\langle g, S_{t / \lambda^{2}} g\right\rangle \frac{d t}{\lambda^{2}}= \\
=\int_{-\infty}^{+\infty} e^{-i \lambda^{2} \omega u}\left\langle g, S_{u} g\right\rangle d u=\hat{G}\left(\lambda^{2} \omega\right)
\end{gathered}
$$

The above choice of $g^{\lambda}$ and the replacement of $S_{t}$ by $S_{t / \lambda^{2}}$ correspond to a total Hamiltonian

$$
H_{\lambda}=H_{S} \otimes 1_{R}+\lambda^{-2} 1_{S} \otimes H_{R}+\lambda^{-1} V
$$

with $V=\frac{1}{i}\left[D \otimes a^{+}(g)-D^{+} \otimes a(g)\right]$ (see eq. (5.47) of [Spohn 1980]).

Let $\tau=\lambda^{2} t$, it is immediately seen that

$$
\exp \left[i \tau H_{\lambda}\right]=\exp \left[i t H_{\lambda}^{(w)}\right]=\exp \left[i\left(\tau / \lambda^{2}\right) H_{\lambda}^{(w)}\right]
$$

where $H_{\lambda}$ is given by (14) and $H_{\lambda}^{(w)}$ is given by

$$
H_{\lambda}^{(w)}=\lambda^{2} H_{S} \otimes 1+1 \otimes H_{R}+\lambda V
$$

This is Theorem 3.1 of [4]. In other words, this means that the modification of the singular coupling limit described above is equivalent to a weak coupling limit with rescaled time (slow macroscopic time $\tau$, fast microscopic time $t, \tau=\lambda^{2} t$ ) and with system Hamiltonian of the order of $\lambda^{2}$. The superscript $(w)$ in (15) stands for weak. In what follows we shall use the scaling (14).

\section{The modified WCL}

One is interested in the convergence as $\lambda \rightarrow 0$ of

$j_{t}^{(\lambda)}(X):=e^{i H_{\lambda} t}(X \otimes 1) e^{-i H_{\lambda} t}=U_{t}^{(\lambda)+}\left(e^{i H_{S} t} X e^{-i H_{S} t} \otimes 1\right) U_{t}^{(\lambda)} ; \quad X \in \mathcal{B}\left(\mathcal{H}_{S}\right)$

where

$$
\left\{\begin{array}{l}
U_{t}^{(\lambda)}=e^{i\left(H_{S} \otimes 1+\frac{1}{\lambda^{2}} \otimes H_{R}\right) t} e^{-i H_{\lambda} t}=u m_{n=0}^{\infty} \int_{0 \leq t_{n} \leq \ldots \leq t_{1} \leq t} u m_{\varepsilon_{1}, d o t s, \varepsilon_{n}=0,1} \\
D_{\varepsilon_{1}}(t) \operatorname{dots} D_{\varepsilon_{n}}\left(t_{n}\right) \otimes A^{\varepsilon_{1}}\left(S_{t_{1} / \lambda^{2}} g\right) \operatorname{dots} A^{\varepsilon_{n}}\left(S_{t_{n} / \lambda^{2}} g\right) d t_{1} \ldots d t_{n}
\end{array}\right.
$$

and by definition:

$$
\left\{\begin{array}{l}
D_{0}(t):=e^{i H_{S} t} D^{+} e^{-i H_{S} t} \\
D_{1}(t):=e^{i H_{S} t} D e^{-i H_{S} t} \\
A^{0}(g):=a(g), \quad A^{1}(g)=a^{+}(g)
\end{array}\right.
$$


If the reference state of the reservoir is the Fock vacuum, then $U_{t}^{(\lambda)}$ should converge to a unitary 1 -parameter family $U(t)$ satisfying

$$
d U(t)=\left[D_{0}(t) d A_{t}-D_{1}(t) d A_{t}^{+}+\hat{G}_{-}(0) D_{0}(t) D_{1}(t) d t\right] U(t)
$$

where $A_{t}, A_{t}^{+}$is the Fock quantum Brownian motion with Ito table:

$$
d A_{t} d A_{t}^{+}=\hat{G}(0) d t, \quad d A_{t}^{+} d A_{t}=0
$$

and

$$
\hat{G}_{-}(0)=\int_{-\infty}^{0}\left\langle g, S_{u} g\right\rangle d u=\frac{1}{2} \hat{G}(0)+\text { imaginary part }
$$

In order to get a feeling of the limiting procedure, it is useful to evaluate the matrix elements, of the first few terms of the iterated series, with respect to some collective coherent vector. For the first term we find:

$$
\begin{gathered}
<u \otimes \Phi_{\lambda}(S, T, f), \lambda \int_{0}^{t / \lambda^{2}} V\left(t_{1}\right) d t_{1} v \otimes \Phi_{\lambda}\left(S^{\prime}, T^{\prime}, f^{\prime}\right)>= \\
=\lambda^{-1} \int_{0}^{t} d t_{1}\left\{<u, e^{i H_{S} t_{1}} D e^{-i H_{S} t_{1}} v><\Phi_{\lambda}(S, T, f), A^{+}\left(S_{t_{1} / \lambda^{2}}^{0} g\right) \Phi_{\lambda}\left(S^{\prime}, T^{\prime}, f^{\prime}\right)>-\right. \\
\left.-<u, e^{i H_{S} t_{1}} D^{+} e^{-i H_{S} t_{1}} v><\Phi_{\lambda}(S, T, f), A\left(S_{t_{1} / \lambda^{2}}^{0} g\right) \Phi_{\lambda}\left(S^{\prime}, T^{\prime}, f^{\prime}\right)>\right\}= \\
=\int_{0}^{t} d t_{1}\left\{<u, D\left(t_{1}\right) v>\left\langle S_{t_{1} / \lambda^{2}}^{0} g, \int_{S / \lambda^{2}}^{T / \lambda^{2}} S_{u}^{0} f\right\rangle d u-\right. \\
\left.-<u, D^{+}\left(t_{1}\right) v>\left\langle\int_{S^{\prime} / \lambda^{2}}^{T^{\prime} / \lambda^{2}} S_{u}^{0} f^{\prime}, S_{t_{1} / \lambda^{2}}^{0} g\right\rangle d u\right\} . \\
\cdot<\Phi_{\lambda}(S, T, f), \Phi_{\lambda}\left(S^{\prime}, T^{\prime}, f^{\prime}\right)>
\end{gathered}
$$

It is clear that as $\lambda \rightarrow 0$, this converges to

$$
\begin{aligned}
\int_{0}^{t} d t_{1}\left\{<u, D\left(t_{1}\right) v>\right. & \left.\chi_{[S, T]}\left(t_{1}\right)(g \mid f)-<u, D^{+}\left(t_{1}\right) v>\chi_{\left[S^{\prime}, T^{\prime}\right]}\left(t_{1}\right)\left(f^{\prime} \mid g\right)\right\} \\
& <\Psi(S, T, f), \Psi\left(S^{\prime}, T^{\prime}, f^{\prime}\right)>
\end{aligned}
$$

which can be rewritten as

$<u \otimes \Psi(S, T, f), \int_{0}^{t}\left\{D\left(t_{1}\right) \otimes d A_{t_{1}}^{+}(g)-D^{+}\left(t_{1}\right) \otimes d A_{t_{1}}(g)\right\} v \otimes \Psi\left(S^{\prime}, T^{\prime}, f^{\prime}\right)>$ 
When $n=2$ one of term corresponding to the case in which the creator and annihilator are used to produce scalar product is equal to

$$
\begin{gathered}
\lambda^{2} \int_{0}^{t / \lambda^{2}} d t_{1} \int^{t_{1}} d t_{2} e^{i t_{1} H_{S} \lambda^{2}} D^{+} e^{-i t_{1} H_{S} \lambda^{2}} e^{i t_{2} H_{S} \lambda^{2}} D e^{-i t_{2} H_{S} \lambda^{2}}\left\langle S_{t_{1}} g, S_{t_{2}} g\right\rangle \\
<\Phi_{\lambda}(S, T, f), \Phi_{\lambda}\left(S^{\prime}, T^{\prime}, f^{\prime}\right)> \\
=\lambda^{-2} \int_{0}^{t} d t_{1} \int_{0}^{t_{1}} d t_{2} e^{i t_{1} H_{S}} D^{+} e^{i\left(t_{2}-t_{1}\right) / \lambda^{2} \cdot \lambda^{2} H_{S}} D e^{-i t_{2} H_{S} \lambda^{2}}\left\langle g, S\left(t_{2}-t_{1}\right) / \lambda^{2} g\right\rangle \cdot \\
\cdot<\Phi_{\lambda}(S, T, f), \Phi_{\lambda}\left(S^{\prime}, T^{\prime}, f^{\prime}\right)> \\
=\int_{0}^{t} d t_{1} \int_{-t_{1} / \lambda^{2}}^{0} d t_{2} e^{i t_{1} H_{S}} D^{+} e^{i t_{2} \lambda^{2} H_{S}} D e^{-i\left(t_{2} \lambda^{2}+t_{1}\right) H_{S}}\left\langle g, S_{t_{2}} g\right\rangle . \\
\cdot<\Phi_{\lambda}(S, T, f), \Phi_{\lambda}\left(S^{\prime}, T^{\prime}, f^{\prime}\right)>
\end{gathered}
$$

which tends to, as $\lambda \rightarrow 0$,

$$
\begin{aligned}
& \int_{0}^{t} e^{i t_{1} H_{S}} D^{+} D e^{-i t_{1} H_{S}}(g \mid g) \cdot<\Psi(S, T, f), \Psi\left(S^{\prime}, T^{\prime}, f^{\prime}\right)>= \\
= & \int_{0}^{t} D^{+}\left(t_{1}\right) D\left(t_{1}\right) d\left[A_{t_{1}}(g), A_{t_{1}}^{+}(g)\right] \cdot<\Psi(S, T, f), \Psi\left(S^{\prime}, T^{\prime}, f^{\prime}\right)>
\end{aligned}
$$

If we define

$$
V_{t}:=e^{-i t H_{S}} U(t)
$$

then

$$
\begin{gathered}
d V_{t}=d\left(e^{-i t H_{S}}\right) U(t)+e^{-i t H_{S}} d U(t)= \\
=-i d t H_{S} V_{t}+\left[D_{0} e^{-i t H_{S}} d A_{t}-D_{1} e^{-i t H_{S}} d A_{t}^{+}+\hat{G}_{-}(0) D_{0} D_{1} e^{-i t H_{S}} d t\right] U(t)= \\
=\left[D_{0} d A_{t}-D_{1} d A_{t}^{+}+\left(\hat{G}_{-}(0) D_{0} D_{1}-i H_{S}\right) d t\right] V_{t}
\end{gathered}
$$

The associated semigroup $T_{t}=\mathbf{E}_{0}\left[j_{t}(X)\right]=\mathbf{E}_{0}\left[V_{t}^{+}(X \otimes 1) V_{1}\right]$ has the generator $L$ determined by

$$
\left\{\begin{array}{l}
L(X)=\hat{G}(0) D^{+} X D-\left(\hat{G}_{-}(0) D^{+} D+i H_{S}\right)^{+} X-X\left(\hat{G}_{-}(0) D^{+} D+i H_{S}\right) \\
=\hat{G}(0)\left(D^{+} X D-\frac{1}{2}\left\{D^{+} D, X\right\}\right)+i\left[H^{\prime}, X\right]
\end{array}\right.
$$

with $H^{\prime}=H_{S}+\left(\operatorname{Im} \hat{G}_{-}(0)\right) D^{+} D$. This form follows from the paper on the singular coupling limit. 
Remark 1 It is very important that $H_{S}$ should appear in the final form of the generator; so use $V_{t}$ and not $U_{t}$.

Remark 2 No assumption should be made on the spectrum of $H_{S}$, but only that $H_{S}$ is bounded (so that $D_{0}(t), D_{1}(t)$ are norm continuous functions of $t$ ). In particular, no assumption should be made as to whether $D_{\varepsilon}(t)$ is a multiple of $D_{\varepsilon}(0)$.

Remark 3 In the special case that $D$ is skew-adjoint, say $D=i Q$, with $Q=Q^{+}$, (so that $D_{\varepsilon}(t)$ cannot be a nontrivial multiple of $D_{\varepsilon}(0)$ ), one obtains

$$
L(X)=-\frac{1}{2} \hat{G}(0)[Q,[Q, X]]+i\left[H^{\prime}, X\right]
$$

Remark 4 We may write

$$
\hat{G}_{-}(0)=\int_{-\infty}^{0}\left\langle g, S_{u} g\right\rangle d u=\int_{-\infty}^{0} d u \int_{-\infty}^{+\infty} d \alpha \frac{e^{i \alpha u}}{2 \pi} \hat{G}(\alpha)=\frac{1}{2} \hat{G}(0)-i \mathcal{P} \int_{-\infty}^{+\infty} \frac{1}{\alpha} \hat{G}(\alpha) d \alpha
$$

where $\mathcal{P}$ denotes the principal part of the integral. In [G \& K, F \& G, 1976] one has $\hat{G}(\alpha)=2 \pi|g(\alpha)|^{2}$ taken to be an even function of $\alpha$ (for this it is essential that $H_{1}$ has a spectrum which is symmetric upon reflection in 0 ) so that $\hat{G}_{-}(0)$ is simply $\frac{1}{2} \hat{G}(0)$. If $\hat{G}(\alpha)$ is a continuous function vanishing on $R_{-}$(as happens if the spectrum of $H_{1}$ is contained in $[0, \infty)$ ) then $\hat{G}(0)=0$ and $\hat{G}_{-}(0)$ is purely imaginary.

Then the singular coupling limit only gives a shift of the Hamiltonian.

\section{BIBLIOGRAPHY}

[1] K.Hepp and E.Lieb, Helv. Phys. Acta 46, 573 (1973))

[2] V.Gorini, A.Kossakowski, J. Math. Phys. 17, 1298 (1976)),

[3] A.Frigerio and V.Gorini, J. Math. Phys. 17, 2123 (1976).

[4] P.F.Palmer, J. Math. Phys. 18, 527 (1976)

[5] H.Spohn, Rev. Mod. Phys. 53,569 (1980).

[6] R.L.Dümcke, J. Math. Phys. 24, 311 (1983)

[7] A.Frigerio and V.Gorini, Commun. Math. Phys. 93, 517 (1984).

[8] A.Frigerio, V.Gorini, A.Kossakowski, E.C.G.Sudarsan, M.Verri, Rep. Math. Phys. Vol. 13 (1978)

[9] L. Accardi, R.Alicki, A.Frigerio, Y.G.Lu: An invitation to the weak coupling and low density limits, to appear in Quantum Probability \& related topics. 
[10] L. Accardi, A.Frigerio, J.T.Lewis, R.I.M.S. Vol. 18, No 1 (1983).

[11] L. Accardi, A.Frigerio, Y.G. Lu: The weak coupling limit as a quantum functional central limit. Commu. Math. Phys. 131, 537-570 (1990). 